\title{
Molecular epidemiology of astrovirus in children with gastroenteritis in southwestern Nigeria
}

\author{
K. O. Arowolo ${ }^{1,2,3}$ (1) C. I. Ayolabi ${ }^{1}$ (I) I. A. Adeleye ${ }^{1} \cdot$ B. Lapinski ${ }^{2} \cdot$ J. S. Santos ${ }^{2} \cdot$ Sonia M. Raboni ${ }^{2}$
}

Received: 26 March 2020 / Accepted: 15 June 2020 / Published online: 8 August 2020

(c) Springer-Verlag GmbH Austria, part of Springer Nature 2020

\begin{abstract}
Human astrovirus (HAstV) is recognized as one of the major causative agents of acute gastroenteritis in children worldwide. Data on the genetic diversity of HAstV in Nigeria are limited. The aim of this study was to determine the prevalence and molecular epidemiology of classical HAstV in children under 5 years of age with acute gastroenteritis in Ogun State, Nigeria. Fecal samples (331) as well as socio-demographic and clinical data were collected across the three senatorial districts of the state from February 2015 to April 2017. One hundred seventy-five samples were randomly selected and analyzed for the presence of HAstV using RT-PCR. PCR amplicons from positive samples were sequenced, and phylogenetic analysis was done to determine genotypes and lineages. The overall prevalence rate was $19.4 \%$ (34), with the highest occurrence observed in 2015 (41.4\%). Viral coinfections were detected in 13 cases (38.2\%). HAstV infection occurred throughout the year and in all age groups, mainly in the age group of 0-12 months. There was significant association between prevalence rate and collection year; however, no association was observed with gender, age, symptoms or risk factors. HAstV-5 was the predominant genotype (76.5\%) circulating throughout the study period, followed by HAstV-1 (23.5\%), which circulated only in the first 2 years of the study. Phylogenetic analysis showed that all HAstV-5 strains detected belonged to the 5a lineage, while HAstV-1 strains were grouped into lineage $1 \mathrm{~b}$. This study, to the best of our knowledge, is the first comprehensive report on molecular characterization of classical HAstV among children with gastroenteritis in the country, and this will serve as baseline information for implementing appropriate infection control practices.
\end{abstract}

\section{Introduction}

Acute gastroenteritis (AGE) has been described globally as the second leading cause of death among children less than 5 years of age [1] with enteric viruses (rotavirus, norovirus, astrovirus, adenovirus) being the recognized major common etiological agents [2].

Astroviruses (AstVs) are small, non-enveloped, singlestranded, positive-sense RNA viruses belonging to the

Handling Editor: Akbar Dastjerdi.

Sonia M. Raboni

sraboni@ufpr.br

1 Department of Microbiology, University of Lagos, Lagos, Nigeria

2 Virology Laboratory, Infectious Diseases Division, Hospital de Clinicas, Universidade Federal do Paraná, Curitiba, Paraná, Brazil

3 Department of Biological Sciences, Lagos State Polytechnic, Ikorodu, Lagos, Nigeria family Astroviridae, which is divided into two genera, Mamastrovirus and Avastrovirus, whose members infect mammals and birds, respectively. The genome, which is 6.8$7.9 \mathrm{~kb}$ long, contains of three open reading frames (ORFs): ORF1a, ORF1b, and ORF2. Both ORF1a and ORF1b encode the non-structural proteins involved in RNA transcription and replication, while ORF2 encodes the capsid protein precursor [3], which exhibits the highest diversity among the viral proteins [4] and is used generally as the basis for genotyping [5].

According to the 2018 report of the ICTV (International Committee on Taxonomy of Viruses) (https://talk.ictvonline .org/taxonomy/), human astroviruses (HAstVs) are classified into classical HAstV, Melbourne (MLB) clade, and Virginia (VA) clade. Classical HAstV comprises genotypes 1-8 (HAstV-1-8) [3]. The MLB and VA clades are highly genetically distinct from the classical HAstVs and are more closely related to animal astroviruses [6].

Transmission of HAstV occurs mainly by the fecal-oral route [3], and its clinical manifestations, lasting 2-4 days, include abdominal pain, vomiting, anorexia, fever, headache, 
diarrhea, and severe dehydration, which leads to hospitalization [7]. These symptoms are usually mild and self-limited in comparison with rotavirus (RV) and norovirus (NoV) infections [8].

The classical HAstVs are circulating globally and associated with $2.9 \%$ to $5.0 \%$ of acute diarrhea in children [9]. Generally, HAstV-1 is the most common genotype found worldwide, followed by genotypes 2 to 5 , which are usually associated with diarrheal outbreaks, whereas HAstV-6 to -8 are seldom detected $[5,10]$. However, the predominant genotype varies with time and location [11].

In some African countries, the prevalence of HAstV in children with acute gastroenteritis has been reported to range from $0.8 \%$ to $9.9 \%$ [12-16]. Also, the prevalence of HAstV among children with acute gastroenteritis in Nigeria has been reported in a few studies [17-21], with only two investigating genetic diversity [20,21].

Therefore, there is a lack of information on the genetic diversity of HAstV, which is important and eventually can be used for vaccine development. The aim of this study was to determine the prevalence and molecular epidemiology of classical HAstV infections in children under 5 years old with acute gastroenteritis in Ogun State, Nigeria.

\section{Materials and methods}

\section{Study population and sample collection}

Stool specimens were collected from 331 hospitalized and general outpatient children $\leq 5$ years of age who presented with acute gastroenteritis of less than or equal to 7 days' duration in three different hospitals across Ogun State: FMCA in Ogun Central (157 children); State Hospital IjebuOde in Ogun East (74 children), and State Hospital Ota in Ogun West (100 children) senatorial districts between February 2015 and April 2017. Diarrhea was defined according to the WHO criteria for children as the passage of three or more loose or liquid stools within 24 hours. Socio-demographic data and clinical information were collected through the use of a standardized questionnaire completed by the parents/guardians of each participant. The Institutional Ethical Committee approved this study, and informed consent was obtained before sample collection. Stool specimens were stored at $-20{ }^{\circ} \mathrm{C}$ until they were processed. One hundred seventy-five fecal samples were randomly selected for analysis.

\section{Sample processing}

Each stool sample (4 to $5 \mathrm{~mL}$ ) was suspended (1:10) in Tris$\mathrm{HCl}(0.01 \mathrm{M})-\mathrm{CaCl}_{2}(0.0015 \mathrm{M})$ buffer and subsequently clarified by centrifugation at 3,000 rpm for 15 minutes.
Thereafter, a 2-mL aliquot of the supernatant was dispensed into sterile cryovials and stored at $-20{ }^{\circ} \mathrm{C}$ until they were transported on dry ice to the Research Laboratory for Molecular Biology and Microbiology, Federal University of Parana (HC-UFPR), Brazil, for analysis.

\section{Nucleic acid extraction and HAstV detection}

Viral nucleic acids were extracted from $200 \mu \mathrm{L}$ of the clarified stool suspension using a High Pure Viral Nucleic Acid Kit (Roche Molecular System Inc., Pleasanton, CA) according to the manufacturer's instructions. The nucleic acid concentration and purity (A260/280) were analyzed by spectrophotometry (NanoDrop 2000c, Thermo Fisher Scientific, Waltham, MA). Eluted nucleic acid was stored at $-80{ }^{\circ} \mathrm{C}$ until analysis.

HAstV detection was performed by reverse transcription polymerase chain reaction (RT-PCR) using a set of specific primers targeting a portion of the ORF2 capsid region, Mon 269 (5'-TCAGATGCATTGTCATTG-3') and Mon 270 (5' CAACTCAGGAAACAGGGAGT-3') [22], and a Platinum ${ }^{\circledR}$ Taq DNA Polymerase Kit (Invitrogen, Carlsbad, CA) in a Veriti ${ }^{\mathrm{TM}}$ 96-well thermal cycler (Applied Biosystems Inc, CA). RT was carried out with random primers using a Superscript III ${ }^{\circledR}$ Reverse Transcriptase Kit (Invitrogen, Carlsbad, CA) according to manufacturer's instructions. Briefly, $2.5 \mu \mathrm{l}$ of cDNA was added to $22.5 \mu \mathrm{l}$ of PCR mix consisting of $2.5 \mu \mathrm{l}$ of $10 \mathrm{x}$ PCR buffer, $0.75 \mu \mathrm{l}$ of $50 \mathrm{mM} \mathrm{MgCl}_{2}$, $0.5 \mu \mathrm{l}$ of dNTPs (10 mM each), $0.5 \mu$ lof primer (10 pmol/ $\mu \mathrm{l}), 0.125 \mu \mathrm{l}$ of Platinum Taq DNA polymerase (200 U/ $\mu$, Invitrogen), and $17.625 \mu \mathrm{l}$ of RNase-free water to a total volume of $25 \mu$ l. The PCR conditions were as follows: $94{ }^{\circ} \mathrm{C}$ for $3 \mathrm{~min}, 40$ cycles of $94{ }^{\circ} \mathrm{C}$ for $30 \mathrm{~s}, 50{ }^{\circ} \mathrm{C}$ for $30 \mathrm{~s}$ and, $72{ }^{\circ} \mathrm{C}$ for $30 \mathrm{~s}$, and final extension at $72{ }^{\circ} \mathrm{C}$ for $2 \mathrm{~min}$. PCR products were resolved by electrophoresis in a $1.5 \%$ agarose gel, stained with ethidium bromide, and viewed using a UV transilluminator, and bands were analyzed using the E-Capt program (Vilber Lourmat Deustchland, GmbH, Germany). Detection of other enteric viruses (group A rotavirus [RVA], norovirus [NoV], and human adenovirus [HAdV]) were also performed simultaneously as described previously [23].

\section{Nucleotide sequencing}

PCR amplicons from positive samples were purified using a QIAquick Gel Extraction Kit (QIAGEN, Germany) according to manufacturer's instructions and sequenced directly in both directions using a Big Dye ${ }^{\circledR}$ Terminator v.3.1 Kit and an ABI 3130 automated sequencer (Applied Biosystems, Foster City, CA, USA) with the same primers used for the amplification reactions. 


\section{Sequence analysis}

Sequencing chromatograms were generated and edited using the SeqMan tool in DNASTAR Lasergene 7.0.0 software (DNASTAR, Inc., Madison, WI, USA). The sequences that were generated were compared with those retrieved from the GenBank database using the Basic Local Alignment Search Tool (BLAST) software, and multiple sequence alignments were performed using ClustalW in MEGA 7 software [24]. The HAstV genotype was inferred by phylogenetic analysis using the maximum-likelihood (ML) method based on the general time-reversible $(\mathrm{GTR}+\mathrm{G}+\mathrm{I})$ substitution model with a bootstrap test of 1000 replicates [25] implemented in the MEGA7 software. Nucleotide sequence identity was calculated using p-distance in MEGA 7 software. The nucleotide sequences determined in this study were submitted to the GenBank database with the accession numbers MN114638-MN114671.

\section{Statistical analysis}

Socio-demographic data and clinical information were compared by chi-square test using IBM SPSS version 20 (IBM Corp, Armonk, NY) for Windows. The level of significance was set at $p<0.05$.

\section{Results}

The overall HAstV detection rate was $19.4 \%$ (34/175). In 2015 , a detection rate of $41.4 \%$ (12/29) was observed in the samples tested, while the detection rate decreased to $16.9 \%(19 / 113)$ and $9.1 \%(3 / 33)$ in 2016 and 2017 , respectively. A significant association was observed between the prevalence of HAstV and the collection year $(p<0.05)$. Considering the different senatorial districts, HAstV prevalence was higher in Ogun East $(25.0 \%, 15 / 60)$ and Ogun West $(23.0 \%, 14 / 61)$ than in Ogun Central $(9.3 \%$ [5/54]) senatorial district. There was no statistically significant association between senatorial districts and the prevalence rate $(p>0.05)$ (Table 1). Coinfection with other tested enteric viruses was seen in $13(38.2 \%)$ of the 34 positive HAstV cases, while $21(61.8 \%)$ were positive only for HAstV. The most commonly detected coinfection was HAstV with RVA (76.9\%, 10/13), followed by HAstV with NoV $(15.4 \%, 2 / 13)$ and HAstV with HAdV (7.7\%, 1/13).

Samples from 99 males and 76 females were analyzed, and the detection rate of HAstV was $19.2 \%(19 / 99)$ and $19.7 \%$ (15/76), respectively. Human astrovirus was detected in all age groups, with the highest prevalence observed among children aged 0-12 months $(23.7 \%$, $14 / 59)$, followed by children aged 13-24 months (20.0\%, 10/50). HAstV was detected in three of the five age groups in 2015, with children 0-12 months having the highest positivity rate of 50\% (6/12 HAstV samples). In 2016, all age groups were infected by HAstV, with children 0-12 and 13-24 months old having the same detection rate of $36.8 \%$ (7/19 HAstV samples), while the 0-12, 37-48, and 49-60 months age groups were only affected in 2017. However, HAstV detection was observed in the 0-12 months age group throughout the study period, and no significant association was observed with respect to gender, age (Table 1), symptoms, or risk factors (data not shown) $(p>0.05)$.

Table 1 Socio-demographic characteristics of HAstV-positive children with gastroenteritis in Ogun State, Nigeria

\begin{tabular}{|c|c|c|c|c|c|c|c|}
\hline & $\begin{array}{l}0-12 \text { months (\%) } \\
\mathrm{n}=14\end{array}$ & $\begin{array}{l}\text { 13-24 months }(\%) \\
\mathrm{n}=10\end{array}$ & $\begin{array}{l}25-36 \text { months }(\%) \\
\mathrm{n}=4\end{array}$ & $\begin{array}{l}37-48 \text { months }(\%) \\
\mathrm{n}=4\end{array}$ & $\begin{array}{l}49-60 \text { months }(\%) \\
\mathrm{n}=2\end{array}$ & Total $(\%)$ & $P$-value \\
\hline \multicolumn{8}{|l|}{ Collection year } \\
\hline $2015(n=29)$ & $6(50)$ & $3(30)$ & $3(75)$ & $0(0)$ & $0(0)$ & $12(41.4)$ & \multirow[t]{3}{*}{0.003} \\
\hline $2016(\mathrm{n}=113)$ & $7(36.8)$ & $7(36.8)$ & $1(5.3)$ & $3(15.8)$ & $1(5.3)$ & $19(16.9)$ & \\
\hline $2017(n=33)$ & $1(33.3)$ & $0(0)$ & $0(0)$ & $1(33.3)$ & $1(33.3)$ & $3(9.1)$ & \\
\hline \multicolumn{8}{|l|}{ Senatorial district } \\
\hline $\begin{array}{l}\text { Ogun Central } \\
(\mathrm{n}=54)\end{array}$ & $0(0)$ & $1(20)$ & $0(0)$ & $2(40)$ & $2(40)$ & $5(9.3)$ & \multirow[t]{3}{*}{0.073} \\
\hline Ogun East $(n=60)$ & $8(13.3)$ & $4(6.7)$ & $3(5.0)$ & $0(0)$ & $0(0)$ & $15(25.0)$ & \\
\hline Ogun West $(n=61)$ & $6(9.8)$ & $5(8.2)$ & $1(1.6)$ & $2(3.3)$ & $0(0)$ & $14(23.0)$ & \\
\hline \multicolumn{8}{|l|}{ Gender } \\
\hline Male (n = 99) & $12(12.1)$ & $1(1.0)$ & $2(2.0)$ & $3(3.0)$ & $1(1.0)$ & $19(19.2)$ & \multirow[t]{2}{*}{0.928} \\
\hline Female $(n=76)$ & $2(2.6)$ & $9(11.8)$ & $2(2.6)$ & $1(1.3)$ & $1(1.3)$ & $15(19.7)$ & \\
\hline
\end{tabular}

Bold print indicates a significant result 
The cumulative highest prevalence of HAstV infection was observed in November $(20.6 \%, 7 / 34)$, with $64.7 \%$ of the total positive cases detected between October and April, corresponding to the dry season of the year. However, HAstV was detected throughout the year except in July and December (Fig. 1).

Sequencing and phylogenetic analysis of a portion of the ORF2 region (449 bp) showed that two different HAstV genotypes (HAstV-1 and HAstV-5) co-circulated in the state. HAstV-5 was the most predominant genotype identified, with a prevalence of $76.5 \%$ (26/34). In the three senatorial districts, HAstV-1 was present in Ogun East and Ogun West senatorial districts at the same rate in 2015 and 2016, but with no occurrence recorded from any of the senatorial districts in 2017. The prevalence of HAstV-1 strains was higher in Ogun East $(62.5 \%, 5 / 8)$ than in Ogun West senatorial district $(37.5 \%, 3 / 8)$. HAstV-5 circulated throughout the sampling period with the highest prevalence in 2016. Ogun West senatorial district had a slightly higher detection rate $(42.3 \%$, 11/26) of HAstV-5 strains than Ogun East (38.5\%, 10/26), and the lowest prevalence (19.2\%) was observed in Ogun
Central senatorial district (Table 2). The age group distribution of HAstV genotypes indicated that both HAstV-1 and HAstV-5 strains mostly affected children up to 2 years of age (Fig. 2).

All eight Nigerian HAstV-1 strains with 100\% nucleotide (nt) sequence identity to one another clustered within lineage $1 \mathrm{~b}$ alongside reference strains detected in China (FJ375759) and Columbia (AF211962), showing 96.3\%96.7\% nt sequence identity. Similarly, HAstV-1b strains reported in this study were closely related to strains from Burkina Faso (LN612582), India (MN242255, KY815036, KY815037), and Lebanon (KX913263) with 98.4\%-98.8\% nt sequence identity. The HAstV-5 strains from this study was also closely related to one another $(99.5 \%-100 \% \mathrm{nt}$ sequence identity), and they clustered within lineage $5 \mathrm{a}$ along with reference strains from the United Kingdom (AB000297), the USA (U15136, MK059953), Ghana (EU327560), Cameroon (MH933758, MH933759), and Burkina Faso (LN612588). They showed 96.3\%-98.4\% nt sequence identity to the HAstV-5a reference strains (Fig. 3).
Fig. 1 Monthly distribution of positive HAstV samples in Ogun State between February 2015 and April 2017
Table 2 Distribution of HAstV genotypes in Ogun State

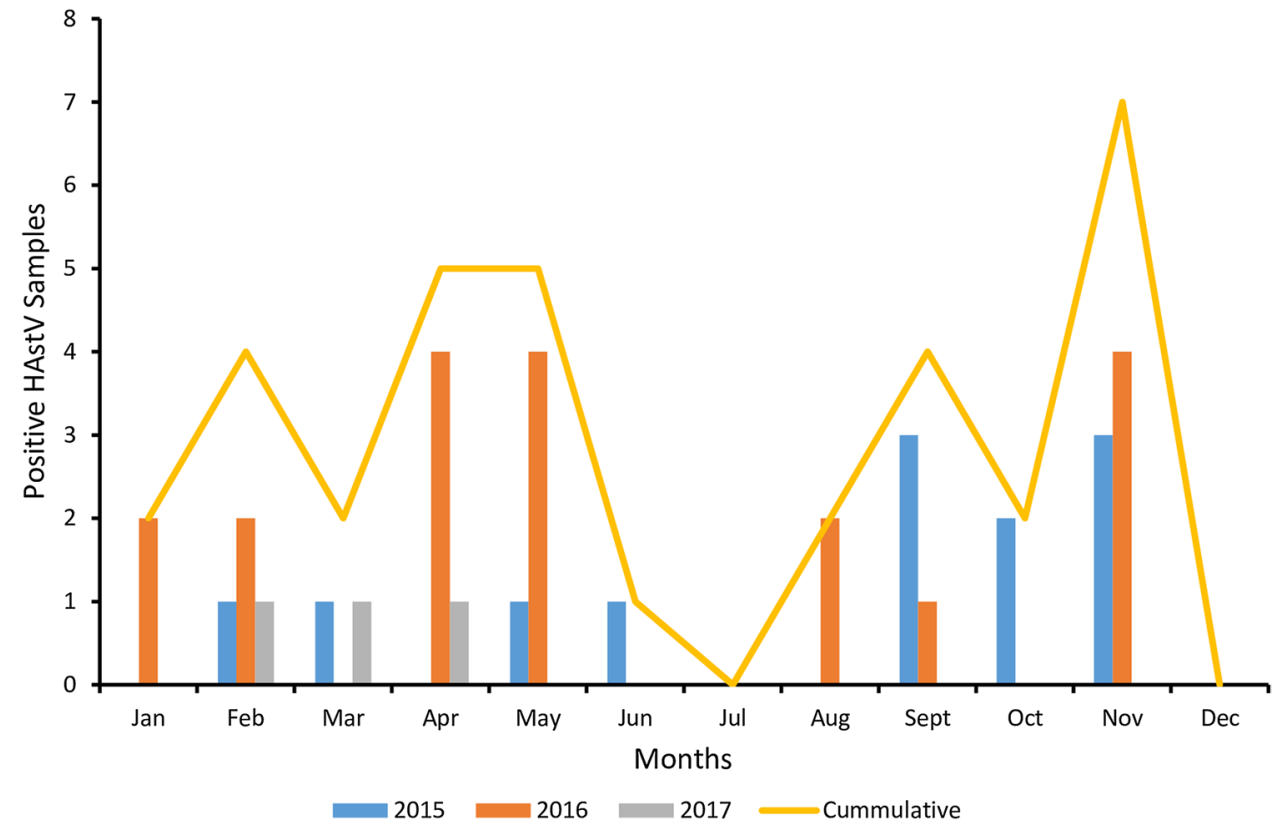

\begin{tabular}{llllllll}
\hline Year & \multicolumn{1}{l}{ HAstV-1 $(\mathrm{n}=8)$} & & \multicolumn{3}{l}{ HAstV-5 $(\mathrm{n}=26)$} \\
& $\begin{array}{l}\text { Ogun Central } \\
\mathrm{n}(\%)\end{array}$ & $\begin{array}{l}\text { Ogun East } \\
\mathrm{n}(\%)\end{array}$ & $\begin{array}{l}\text { Ogun West } \\
\mathrm{n}(\%)\end{array}$ & & $\begin{array}{l}\text { Ogun Central } \\
\mathrm{n}(\%)\end{array}$ & $\begin{array}{l}\text { Ogun East } \\
\mathrm{n}(\%)\end{array}$ & $\begin{array}{l}\text { Ogun West } \\
\mathrm{n}(\%)\end{array}$ \\
\hline 2015 & $0(0)$ & $4(50)$ & $0(0)$ & & $0(0)$ & $6(23.1)$ & $2(7.7)$ \\
2016 & $0(0)$ & $1(12.5)$ & $3(37.5)$ & & $4(15.4)$ & $3(11.5)$ & $8(30.8)$ \\
2017 & $0(0)$ & $0(0)$ & $0(0)$ & & $1(3.8)$ & $1(3.8)$ & $1(3.8)$ \\
Total & $0(0)$ & $5(62.5)$ & $3(37.5)$ & & $5(19.2)$ & $10(38.5)$ & $11(42.3)$ \\
\hline
\end{tabular}


Fig. 2 Age group distribution of HAstV genotypes in Ogun State between February 2015 and April 2017

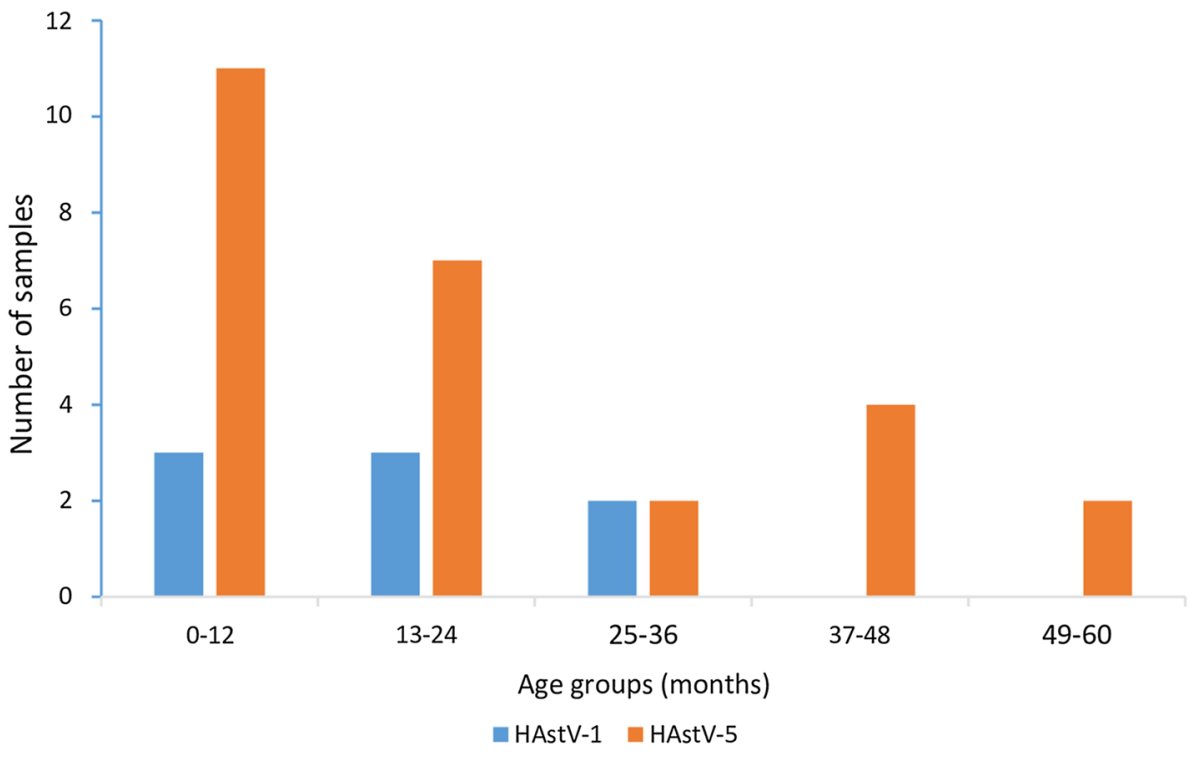

\section{Discussion}

This study, to the best of our knowledge, is the first comprehensive phylogenetic analysis of classical HAstVs in South West Nigeria. The overall high HAstV prevalence rate $(19.4 \%)$ observed in this study is in contrast to the global average incidence of $11 \%$ [3] as well as to those reported previously in Nigeria [17, 19-21] and other countries, including Tunisia [15], South Africa [16], Gabon [26], Lebanon [27], Germany [28], Korea [29], and Brazil [30]. However, this prevalence is lower than that reported previously in Lagos, Nigeria (40.4\%) [18]. However, both had higher prevalence when compared to other Nigerian studies, suggesting that $\mathrm{HAst} \mathrm{V}$ is contributing significantly to the burden of gastroenteritis in children in Ogun State, Nigeria. The discrepancies in prevalence rates may be due to sample size, study population, participants' age, geographical location, climatic factors, and different diagnostic methods used in various studies.

A relatively low frequency of coinfection $(38.2 \%)$ with other enteric viruses was observed in this study, which is lower than previously reported in Osun State, Nigeria (42.9\%) [21], Germany (71\%) [28], Bangladesh (77\%) [31], and Italy (50\%) [32]. This difference may be due to viral detection methods, sample size, period of study, and indistinct seasonality. The predominant coinfection was HAstV with RVA, which has also been reported in Uruguay [11] and Italy [32], but this is in contrast with reports by Japhet et al. [21], Ouédraogo et al. [33], and Sequeira et al. [34] who detected RVA with NoV, RVA with HAdV, and HAstV with $\mathrm{NoV}$, respectively. The results of our study imply that HAstV and RVA coinfection occurs frequently in AGE children, which contrast with the report by Sequeira et al. [34], who suggested that this is a rare occurrence.
The detection rate of HAstV in 2015 (41.4\%) is higher than in studies from Osun State in 2012-2013 (6.8\%) [21] and North East Nigeria in 2013-2014 (5\%) [20]. Similarly, a molecular epidemiology study from Korea from 2013 to 2017 revealed a $2.1 \%$ positive rate in 2015 [29]. This implies that $\mathrm{HAstV}$ occurrence may vary substantially over the years within the same or different locations/countries. There was a significant association between prevalence rate and collection year, which might be a consequence of a smaller number of samples collected in 2015 or the smaller number of positive samples observed in 2015 and 2017.

Human astrovirus prevalence was lower in Ogun Central senatorial district than in the other districts, which might be due to the higher level of urbanization and socio-economic status of people living in the capital city of the state where the senatorial district is located. Consistent with findings from Nigeria [18, 19] and Lebanon [27], the burden of HAstV infection did not vary significantly between males and females.

The occurrence of HAstV infection was mainly in the dry season, which is in contrast to reports by Maldonado et al. [35] and Bosch et al. [36] who found that the maximum incidence of HAstV infections in the tropical areas tends to occur in the rainy season. This discrepancy may be a result of climatic conditions, geographical location, and sampling period. However, the finding of this study is similar to a report from Burkina Faso [37]. In other studies [16, $38,39]$, HAstV was detected throughout the year, although Guix et al. [5] in Spain observed a major peak in November, which agrees with the findings of this study.

Our study revealed the genotypic variability of classical HAstV with two genotypes co-circulating in Nigeria. In contrast with studies from several regions of the world reporting a high frequency of HAstV-1 [31, 33, 40, 41], HAstV-5 was 
Fig. 3 Phylogenetic analysis based on partial ORF2 sequences of classical HAstVs detected in children with acute gastroenteritis in Ogun State, Nigeria, from February 2015 to April 2017. The tree was inferred using the maximumlikelihood (ML) method with bootstrap support values $>70$ (1000 replicates) shown on the left of the nodes. The strains from this study are indicated by diamonds, and HAstV reference strains obtained from the GenBank database are indicated by accession number, isolate, country, and year. The scale bar represents nucleotide substitution per site

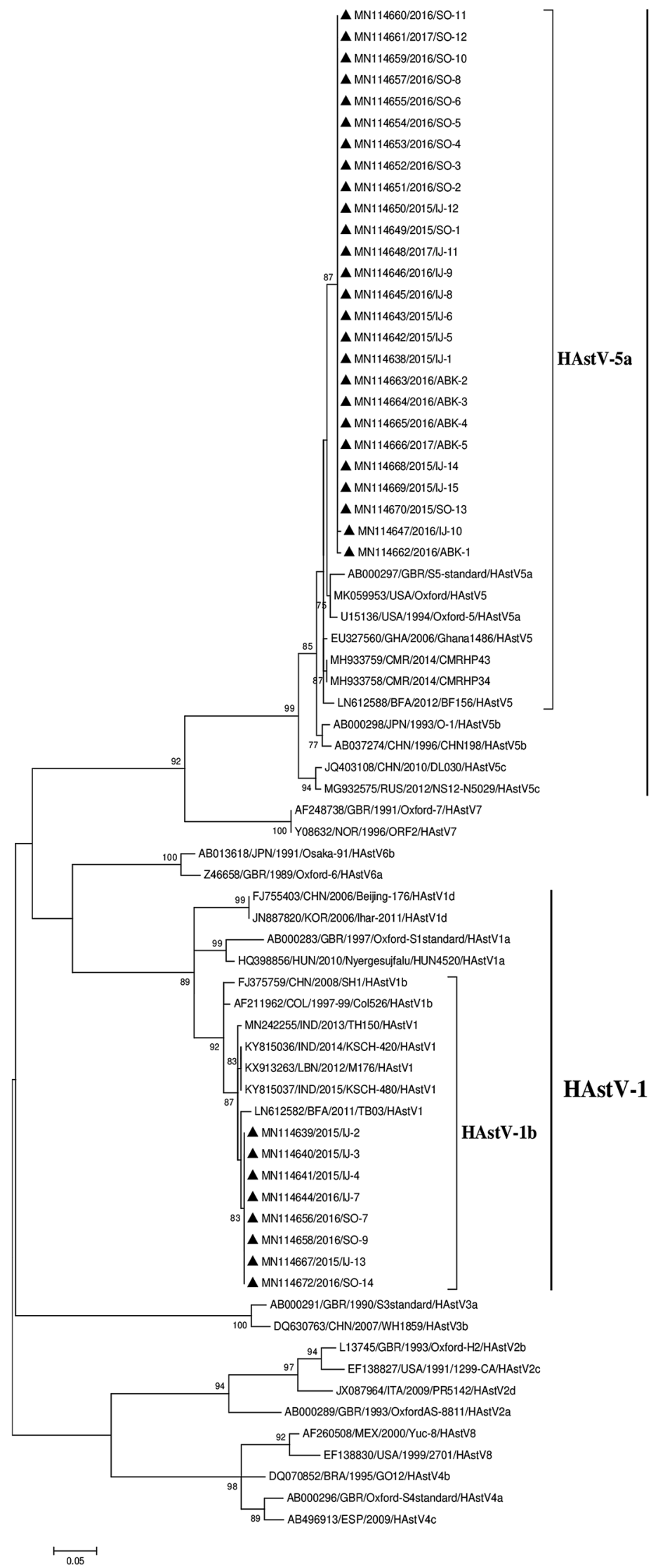

HAstV-5 
the predominant genotype (76.5\%) in this study, which is a rare occurrence in HAstV studies. In addition, HAstV-5 have been reported at low frequency in North East Nigeria (16.7\%) [20], Burkina Faso (8.33\%) [33], South Africa (9\%) [42], and Egypt (15.7\%) [43]. HAstV-5 was detected throughout the study period, which is similar to a study from North East Nigeria [20] in which HAstV-5 was the only genotype detected. This could imply that this genotype is the most dominant in Nigeria. Ogun West senatorial district had a higher detection rate of $\mathrm{HAstV}-5$, which might be a result of its proximity to the Republic of Benin, which serves as an access route to Economic Community of West African States (ECOWAS) markets, thereby increasing the movement of people in and out of the senatorial district as well as the transmission of the virus.

The HAstV genotypes in this study predominantly affected children in the age group of $\leq 2$ years, which is consistent with reports by De Benedictis et al. [7] and Bosch et al. [36] but differs from the study by Lopez et al. [11]. Also, HAstV infection was mainly detected in children 0-12 months of age, which is similar to what has been observed in Nigeria [18, 19], Gabon [26], Lebanon [27], and the cities of Belem [34] and Rio Branco [44] in northern Brazil. However, the age distribution of HAstV infection was not statistically significant, but HAstV was detected in all other age groups analyzed, which is in agreement with reports by Kuta et al. [19], Lopez et al. [11] and Jacobsen et al. [28]. This implies that HAstV infection can occur at different ages between 0 and 5 years, suggesting that HAstV vaccines should be administered to children early in life to confer immunity.

The HAstV-5 strains in this study were classified as lineage $5 \mathrm{a}$, and to the best of our knowledge, this is the first and only detection of this lineage in Nigeria, and other lineages were not detected. The sequences from the study conducted in North East Nigeria [20] were unavailable from GenBank for phylogenetic analysis, while the one reported in Osun State, Nigeria was from a region (ORF1b) [21] different from the target region used in this study (ORF2). The phylogenetic tree shows that the reference strains (AB000297, MK059953 and U15136) are probably evolutionary ancestors of the Nigerian HAstV-5a strains. However, the African strains that clustered within the 5a lineage are all from sub-Saharan African countries, including Nigeria, which might suggest that HAstV-5a strains are circulating within the region. Therefore, the HAstV-5a strains could be said to have shown geographical clustering with possible impact on epidemiology.

HAstV-1 was less frequently detected in this study in contrast to studies from Uruguay [11], Lebanon [27], Germany [28], Korea [29], northern Brazil [44], and Burkina Faso [33] but similar to studies by Guerrero et al. in Mexico City [45] and Morillo et al. in Brazil [30]. This disparity might be as a result of geographical location and temporal distribution. HAstV-1 was detected only at low rates in 2015 and 2016 compared to HAstV-5, which suggests that HAstV-1 is just emerging or that HAstV-5 might have replaced genotype 1 over time, which would explain the high prevalence of genotype 5 in the study. Ogun East senatorial district had a higher HAstV-1 prevalence rate than Ogun West senatorial district. This is most likely due to chance, as both districts had almost the same number of analyzed samples. The HAstV-1 strains in this study were all grouped into lineage $1 \mathrm{~b}$, whereas lineage $1 \mathrm{a}$ was observed in previous studies by Morillo et al. [30], Siqueira et al. [34], Bitencurt et al. [44], as well as Medina et al. [46] and Guix et al. [5], who identified more than one lineage. This is the first time HAstV- $1 \mathrm{~b}$ is being detected among gastroenteritis children in Nigeria. However, HAstV-1 was reported in children with non-polio acute flaccid paralysis in Nigeria in a study targeting the ORF1b region by Kapoor et al. [47]. In this study, the Nigerian HAstV-1b strains clustered with strains from different countries with no distinct geographical clustering, and introduction of strains is continuous, which has a possible impact on epidemiology.

The limitations of this study include (i) partial nucleotide sequencing of the ORF2 region, which may have effect on the entire phylogenetic analysis, and the use of only classical HAstV primers for detection, which prevents novel AstV epidemiology in the study population, and (ii) the lack of use of a community-based study design. However, representative hospitals covering the three senatorial districts of the state were used.

In conclusion, this study shows that HAstV is contributing substantially to the burden of gastroenteritis and is detected in all age groups between 0 and 5 years, with the highest prevalence in 0- to 12-month-old children. HAstV infection occurred throughout the year (except July and December), with a high prevalence being recorded mainly in the dry season. There was co-circulation of two distinct HAstV genotypes (HAstV-5a and HAstV-1b), with the former being predominant. This is the first comprehensive study on the genetic diversity of classical HAstVs in Ogun State, South West Nigeria, which will provide baseline epidemiological information for the future formulation and application of an effective HAstV vaccine.

Acknowledgements The authors acknowledge all the staff of the pediatric clinic of the selected hospitals for their assistance, the field workers, and the children/parents (guardians) who participated in this study.

Author contributions KOA, CIA and IAA conceived and designed the study. KOA collected the samples. KOA, CIA, BL, JSS, and SMR developed the methodology. KOA, BL and JSS performed the experiments. KOA analyzed, interpreted the data, and drafted the manuscript. KOA, CIA, IAA, JSS and SMR revised the manuscript. All authors read and approved the final manuscript. SMR and CIA supervised this study. 


\section{Compliance with ethical standards}

Funding None.

Conflict of interest The authors declare that they have no conflict of interest.

Ethical approval This study was approved by the Research Ethics Committee of Federal Medical Centre Abeokuta (FMCA) (FMCA/470/ HREC/09/2015) as well as State Hospital, Ijebu-Ode (RER/AP/ VOL/113) and Ota (2265/02). Written informed consent was obtained from the parents/guardians of all participants included in the study.

\section{References}

1. Liu L, Oza S, Hogan D et al (2016) Global, regional, and national causes of under 5 mortality in 2000-15: an updated systematic analysis with implications for the sustainable development goals. Lancet 388:3027-3035

2. Grytdal SP, DeBess E, Lee LE, Biggs C et al (2016) Incidence of norovirus and other viral pathogens that cause acute gastroenteritis (AGE) among Kaiser Permanente Member populations in the United States, 2012-2013. PLoS One 11:e0148395

3. Monroe SS, Jiang B, Stine SE, Koopmans M, Glass RI (1993) Subgenomic RNA sequence of human astrovirus supports classification of Astroviridae as a new family of RNA viruses. J Virol 67:3611-3614

4. Strain E, Kelley LA, Schultz-Cherry S, Muse SV, Koci MD (2008) Genomic analysis of closely related astroviruses. J Virol 82:5099-5103

5. Guix S, Caballero S, Villena C et al (2002) Molecular epidemiology of astrovirus infection in Barcelona, Spain. J Clin Microbiol 40:133-139

6. Hata A, Katayama H, Kitajima M, Furumai H (2015) Wastewater analysis indicates that genetically diverse astroviruses, including strains belonging to novel clades MLB and VA, are circulating within Japanese populations. Appl Environ Microbiol 81:4932-4939

7. De Benedictis P, Schultz-Cherry S, Burnham A, Cattoli G (2011) Astrovirus infections in humans and animals-molecular biology genetic diversity, and interspecies transmissions. Infect Genet Evol 11:1529-1544

8. Cortez V, Freiden P, Gu Z, Adderson E, Hayden R, SchultzCherry S (2017) Persistent infections with diverse co-circulating astroviruses in pediatric oncology patients, Memphis, Tennessee, USA. Emerg Infect Dis 23(2):288

9. Vu D-L, Bosch A, Pintó RM, Guix S (2017) Epidemiology of classic and novel human astrovirus: gastroenteritis and beyond. Viruses 9:33

10. Gabbay YB, Leite JP, Oliveira DS, Nakamura LS, NunesMR Mascarenhas JD, Heinemann MB, Linhares AC (2007) Molecular epidemiology of astrovirus type 1 in Belem, Brazil, as an agent of infantile gastroenteritis, over a period of 18 years (1982-2000): identification of two possible new lineages. Virus Res 129:166-174

11. Lopez F, Lizasoain A, Victoria M, Papalardo C, Castro S, Arreseigor E, López P, Colina R (2017) Epidemiology and genetic diversity of classic human astrovirus among hospitalized children with acute gastroenteritis in Uruguay. J Med Virol 89:1775-1781

12. Phan TG, Nordgren J, Ouermi D, Simpore J, Nitiema LW, Deng $X$ et al (2014) New astrovirus in human feces from Burkina Faso. J Clin Virol 60:161-164
13. Meyer CT, Bauer IK, Antonio M, Adeyemi M, Saha D, Oundo JO et al (2015) Prevalence of classic, MLB-clade and VA-clade Astroviruses in Kenya and The Gambia. Virol J 12:78

14. Liu J, Platts-Mills JA, Juma J, Kabir F, Nkeze J, Okoi C et al (2016) Use of quantitative molecular diagnostic methods to identify causes of diarrhoea in children: a re-analysis of the GEMS case-control study. Lancet 388:1291-1301

15. Monastiri A, Aouni M, Guix S, Mechri B, Lopez-Roig M, Abid NBS et al (2016) Clinical surveillance for human astrovirus in Monastir region, Tunisia. BMC Public Health 16:57

16. Nadan S, Taylor MB, Groome MJ, Cohen C, Madhi SA, Page NA (2019) Epidemiology of human astrovirus among children younger than 5 years: prospective hospital-based sentinel surveillance in South Africa, 2009-2014. J Med Virol 91:225-234

17. Aminu M, Esona MD, Geyer A, Steele AD (2008) Epidemiology of rotavirus and astrovirus Infections in children in northwestern Nigeria. Ann Afr Med 7:168-174

18. Ayolabi C, Ojo D, Akpan I (2012) Astrovirus infection in children in lagos, Nigeria. Afr J Infect Dis 6:1-4

19. Kuta FA, Damisa D, Adabara NU, Abdulsalam R (2014) Prevalence of astrovirus infection in children in Nasarawa state, Nigeria. GARJM 3:102-105

20. Oyinloye SO, Aminu M, Ella EE, Nimzing L (2018) Molecular detection of astrovirus in diarrhoeic stools of children in North East Nigeria. MRJI 26:1-10

21. Japhet MO, Famurewa O, Adesina OA et al (2019) Viral gastroenteritis among children of $0-5$ years in Nigeria: Characterization of the first Nigerian aichivirus, recombinant noroviruses and detection of a zoonotic astrovirus. JCV 111:4-11

22. Noel JS, Lee TW, Kurtz JB, Glass RI, Monroe SS (1995) Typng of human astroviruses from clinical isolates by enzyme immunoassay and nucleotide sequencing. J Clin Microbiol 33:797-801

23. Arowolo KO, Ayolabi CI, Lapinski B, Santos JS, Raboni SM (2019) Epidemiology of enteric viruses in children with gastroenteritis in Ogun State, Nigeria. J Med Virol 91:1022-1029

24. Kumar S, Stecher G, Tamura K (2016) MEGA7: Molecular Evolutionary Genetics Analysis Version 7.0 for Bigger Datasets. Mol Biol Evol 33:1870-1874

25. Felsenstein J (1985) Confidence limits on phylogenies: an approach using the bootstrap. Evolution 39:783-791

26. Lekana-Douki SE, Kombila-Koumavor C, Nkoghe D, Drosten C, Drexler JF, Leroy EM (2015) Molecular epidemiology of enteric viruses and genotyping of rotavirus $\mathrm{A}$, adenovirus and astrovirus among children under 5 years old in Gabon. Int J Infect Dis 34:90-95

27. Zaraket H, Abou-El-Hassan H, Kreidieh K et al (2017) Characterization of astrovirus-associated gastroenteritis in hospitalized children under five years of age. Infect Genet Evol 53:94-99

28. Jacobsen S, Höhne M, Marques AM, Beslmüller K, Bock CT, Niendorf S (2018) Co-circulation of classic and novel astrovirus strains in patients with acute gastroenteritis in Germany. J Infect 76:457-464

29. Kim JS, Lee WJ, Lee SK et al (2019) Molecular epidemiology of human astrovirus in stool samples from patients with acute gastroenteritis in Korea, 2013-2017. Ann Lab Med 39:367-372

30. Morillo SG, Luchs A, Cilli A, Carmona RCC, Timenetsky MDCST (2018) Detection and genetic characterization of classic human astroviruses in Brazil, 2010-2012. Arch Virol 63:1293-1297

31. Afrad MH, Karmakar PC, Das SK et al (2013) Epidemiology and genetic diversity of human astrovirus infection among hospitalized patients with acute diarrhea in Bangladesh from 2010 to 2012. J Clin Virol 58:612-618

32. De Grazia S, Platia MA, Rotolo V, Colomba C, Martella V, Glammanco GM (2011) Surveillance of human astrovirus circulation in 
Italy 2002-2005: emergence of lineage 2c strains. Clin Microbiol Infect 17:97-101

33. Ouédraogo N, Kaplon J, Bonkoungou IJO et al (2016) Prevalence and genetic diversity of enteric viruses in children with diarrhea in Ouagadougou, Burkina Faso. PLoS One 11:e0153652

34. Siqueira JAM, Oliveira DS, Carvalho TCN et al (2017) Astrovirus infection in hospitalized children: molecular, clinical and epidemiological features. J Clin Virol 94:79-85

35. Maldonado Y, Cantwell M, Old M et al (1998) Population-based prevalence of symptomatic and asymptomatic astrovirus infection in rural Mayan infants. J Infect Dis 178:334-339

36. Bosch A, Pintó RM, Guix S (2014) Human astroviruses. Clin Microbiol Rev 27:1048-1074

37. Ouedraogo N, Ngangas SMT, Bonkoungou IJO et al (2017) Temporal distribution of gastroenteritis viruses in Ouagadougou, Burkina Faso: seasonality of rotavirus. BMC Public Health 17:274

38. Platts-Mills JA, Babji S, Bodhidatta L et al (2015) Pathogen-specific burdens of community diarrhoea in developing countries: a multisite birth cohort study (MAL-ED). Lancet Glob Health 3:e564-e575

39. Shioda K, Cosmas L, Audi A et al (2016) Population-based incidence rates of diarrheal disease associated with norovirus, sapovirus, and astrovirus in Kenya. PLoS One 11(4):e0145943

40. Chhabra P, Payne DC, Szilagyi PG et al (2013) Etiology of viral gastroenteritis in children $<5$ years of age in the United States, 2008-2009. J Infect Dis 208:790-800

41. Nakamura N, Kobayashi S, Minagawa H, Matsushita T, Sugiura W, Iwatani Y (2016) Molecular epidemiology of enteric viruses in patients with acute gastroenteritis in Aichi prefecture, Japan, 2008/09-2013/14. J Med Virol 88:1180-1186

42. Nadan S, Walter JE, Grabow WOK, Mitchell DK, Taylor MB (2003) Molecular characterization of astroviruses by reverse transcriptase PCR and sequence analysis: comparison of clinical and environmental isolates from South Africa. Appl Environ Microbiol 69:747-753

43. Naficy AB, Rao MR, Holmes JL et al (2000) Astrovirus diarrhea in Egyptian children. J Infect Dis 182:685-690

44. Bitencurt ELR, Siqueira JAM, Medeiros TB et al (2019) Epidemiological and molecular investigation of norovirus and astrovirus infections in Rio Branco, Acre, Northern Brazil: a retrospective study. J Med Virol 91:997-1007

45. Guerrero ML, Noel JS, Mitchell DK et al (1998) A prospective study of astrovirus diarrhea of infancy in Mexico City. Pediatr J Infect Dis 17:723-727

46. Medina SM, Gutierrez MF, Liprandi F, Ludert JE (2000) Identification and type distribution of astroviruses among children with gastroenteritis in Colombia and Venezuela. J Clin Microbiol 38:3481-3483

47. Kapoor A, Li L, Victoria J et al (2009) Multiple novel astrovirus species in human stool. J Gen Virol 90:2965-2972

Publisher's Note Springer Nature remains neutral with regard to jurisdictional claims in published maps and institutional affiliations. 